\section{Correction: CATALISE: A Multinational and Multidisciplinary Delphi Consensus Study. Identifying Language Impairments in Children}

\section{The PLOS ONE Staff}

\section{Notice of Republication}

This article was republished on September 6, 2016, to correct errors in formatting that were introduced during the typesetting process, as well as correct an error in Table 1. The publisher apologizes for the errors. Please download this article again to view the correct version. The originally published, uncorrected article and the republished, corrected article are provided here for reference.

\section{Supporting Information}

S1 File. Originally published, uncorrected article. (PDF)

S2 File. Republished, corrected article.

(PDF)

\section{Reference}

1. Bishop DVM, Snowling MJ, Thompson PA, Greenhalgh T, CATALISE consortium (2016) CATALISE: A Multinational and Multidisciplinary Delphi Consensus Study. Identifying Language Impairments in Children. PLoS ONE 11(7): e0158753. doi: 10.1371/journal.pone.0158753 PMID: 27392128

\section{G openaccess}

Citation: The PLOS ONE Staff (2016) Correction: CATALISE: A Multinational and Multidisciplinary Delphi Consensus Study. Identifying Language Impairments in Children. PLOS ONE 11(12): e0168066. doi:10.1371/journal.pone.0168066

Published: December 9, 2016

Copyright: @ 2016 The PLOS ONE Staff. This is an open access article distributed under the terms of the Creative Commons Attribution License, which permits unrestricted use, distribution, and reproduction in any medium, provided the original author and source are credited. 